INPLASY

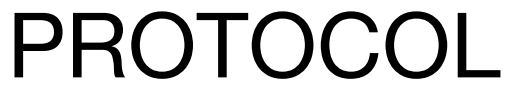

To cite: Zhou et al.

Effectiveness of acupuncture

for Asthenozoospermia: a

protocol for systematic review and meta-analysis. Inplasy protocol 202140032. doi:

10.37766/inplasy2021.4.0032

Received: 05 April 2021

Published: 06 April 2021

Corresponding author:

Shenghui Chen

826994938@qq.com

Author Affiliation:

Nanchang Reproductive

Hospital

Support: Grant No.2020.9.

Review Stage at time of this submission: The review has not yet started.

Conflicts of interest:

None declared.

\section{Effectiveness of acupuncture for Asthenozoospermia: a protocol for systematic review and meta-analysis}

Zhou, YL1; Yao, WL2; Zhang, DJ3; Yu, YL4; Chen, SH5; Lu, HY6; Jiang, $\mathrm{CR}^{7}$; Jiang, $\mathrm{CR}^{8}$.

Review question / Objective: 1. Type of studies. All RCTs which compared acupuncture with either placebo or other drugs. RCTs conducted in adults (participants aged $>18$ years) without regional and language restrictions. 2. Type of participants. Males diagnosed with asthenozoospermia will be considered. The diagnostic criteria for oligoasthenozoospermia, as defined by the WHO laboratory standard, are as follows: sperm concentration $<15 \times 106 / \mathrm{ml}$ and sperm motility (grade A) $<32 \%$ (WHO, 2010). 3. Type of interventions. The experimental group is defined as acupuncture treatment, such as manual acupuncture, warm needling moxibustion, electro-acupuncture, auricular acupuncture, fire needling, elongated needle or moxibustion. 4. Type of comparators. The control group that will include non-acupuncture techniques, such as sham acupuncture, placebo, adjuvant chemotherapy or other pharmacotherapy. The acupoint numbers, retaining time and frequency will not be restricted in this protocol. 5. Types of outcome measures. Primary outcomes will be the total effective rate evaluated by grade $A$ and $B$ sperm. Secondary outcomes will be Sperm motility, sperm DNA fragmentation index (DFI), pregnancy rate and the rate of adverse effects (AEs).

INPLASY registration number: This protocol was registered with the International Platform of Registered Systematic Review and Meta-Analysis Protocols (INPLASY) on 06 April 2021 and was last updated on 06 April 2021 (registration number INPLASY202140032).

\section{INTRODUCTION}

Review question / Objective: 1. Type of studies. All RCTs which compared acupuncture with either placebo or other drugs. RCTs conducted in adults (participants aged $>18$ years) without regional and language restrictions. 2. Type of participants. Males diagnosed with asthenozoospermia will be considered. The 
diagnostic criteria for oligoasthenozoospermia, as defined by the WHO laboratory standard, are as follows: sperm concentration $<15 \times 106 / \mathrm{ml}$ and sperm motility (grade A) <32\% (WHO, 2010). 3. Type of interventions. The experimental group is defined as acupuncture treatment, such as manual acupuncture, warm needling moxibustion, electro-acupuncture, auricular acupuncture, fire needling, elongated needle or moxibustion. 4. Type of comparators. The control group that will include non-acupuncture techniques, such as sham acupuncture, placebo, adjuvant chemotherapy or other pharmacotherapy. The acupoint numbers, retaining time and frequency will not be restricted in this protocol. 5. Types of outcome measures. Primary outcomes will be the total effective rate evaluated by grade $A$ and $B$ sperm. Secondary outcomes will be Sperm motility, sperm DNA fragmentation index (DFI), pregnancy rate and the rate of adverse effects (AEs).

Condition being studied: According to the World Health Organization, the global incidence of infertility is about $15 \%$, and more than $50 \%$ of infertility cases are caused by male infertility. Asthenospermia is caused by male fertility decline and male infertility. Due to work pressure, environmental pollution, sexual diseases and other factors, the number of patients with asthenospermia has increased in recent years. It has been confirmed that acupuncture has a certain effect on patients with asthenospermia. Acupuncture and moxibustion can be an adjuvant treatment plan for the treatment of asthenospermia in addition to drug treatment.

\section{METHODS}

Participant or population: All RCTs which compared acupuncture with either placebo or other drugs. RCTs conducted in adults (participants aged > 18 years) without regional and language restrictions.

Intervention: The experimental group is defined as acupuncture treatment, such as manual acupuncture, warm needling moxibustion, electro-acupuncture, auricular acupuncture, fire needling, elongated needle or moxibustion.

Comparator: The control group that will include non-acupuncture techniques, such as sham acupuncture, placebo, adjuvant chemotherapy or other pharmacotherapy. The acupoint numbers, retaining time and frequency will not be restricted in this protocol.

Study designs to be included: Primary outcomes will be the total effective rate evaluated by grade A and B sperm. Secondary outcomes will be Sperm motility, sperm DNA fragmentation index (DFI), pregnancy rate and the rate of adverse effects (AEs).

Eligibility criteria: 1 . Type of studies.All RCTs which compared acupuncture with either placebo or other drugs. RCTs conducted in adults (participants aged $>18$ years) without regional and language restrictions. 2. Type of participants.Males diagnosed with asthenozoospermia will be considered. The diagnostic criteria for oligoasthenozoospermia, as defined by the WHO laboratory standard, are as follows: sperm concentration $<15 \times 106 / \mathrm{ml}$ and sperm motility (grade A) $<32 \%$ (WHO, 2010).3. Type of interventions. The experimental group is defined as acupuncture treatment, such as manual acupuncture, warm needling moxibustion, electro-acupuncture, auricular acupuncture, fire needling, elongated needle or moxibustion. 4. Type of comparators. The control group that will include non-acupuncture techniques, such as sham acupuncture, placebo, adjuvant chemotherapy or other pharmacotherapy. The acupoint numbers, retaining time and frequency will not be restricted in this protocol. 5. Types of outcome measures. Primary outcomes will be the total effective rate evaluated by grade A and B sperm. Secondary outcomes will be Sperm motility, sperm DNA fragmentation index (DFI), pregnancy rate and the rate of adverse effects (AEs). 
Information sources: The electronic databases of MEDLINE, EMBASE, Cochrane Library, China National Knowledge Infrastructure Database (CNKI), Wan fang Database, China Biology Medicine Database (CBM), VIP Science Technology Periodical Database will be retrieved. We will also manually search unpublished studies and references.

Main outcome(s): The total effective rate evaluated by grade $A$ and $B$ sperm.

Additional outcome(s): Sperm motility, sperm DNA fragmentation index (DFI), pregnancy rate and the rate of adverse effects (AEs).

Quality assessment / Risk of bias analysis: The Cochrane risk assessment tool will be used by us to evaluate the methodological quality of qualified RCTs. It includes 7 items: random sequence generation, allocation concealment, blinding of participants and caregivers, blinding of outcome assessors, incomplete outcome data, selective outcome reporting and other bias. The evaluation result of each item will be "high risk", "low risk", or "unclear risk". The assessment will be completed by 2 reviewers, and disagreements will be handed over to the third reviewer for the final decision.

Strategy of data synthesis: Data synthesis will be completed using RevMan5.3.5 software. When $12<50 \%$, we will choose the fixed effects model; Otherwise, the random effects model will be selected. The forest plots will present the results of the meta-analyses. We will conduct descriptive analysis, when the results are notsuitable for consolidation. When more than 10 studies are included, we will use the funnel plot to assess publication bias.

Subgroup analysis: If there is significant heterogeneity between the study results, we will perform a subgroup analysis to investigate differences in gender, age, outcome styles, etc.

Sensitivity analysis: We will perform sensitivity analyses to verify robustness of results. It includes the impact of methodological quality, study design and sample size.

Country(ies) involved: Nanchang, China.

Keywords: Asthenozoospermia; acupuncture; meta-analysis; male infertility.

Contributions of each author:

Author 1 - Yuliang Zhou.

Email: 469679387@qq.com

Author 2 - Wenliang Yao.

Email: 475644902@qq.com

Author 3 - Duanjun Zhang.

Email: 1148343119@qq.com

Author 4 - Yinglv Yu.

Email: 2649697930@qq.com

Author 5 - Shenghui Chen.

Email: 826994938@qq.com

Author 6 - Huiyu Lu.

Email: 1095779183@qq.com

Author 7 - Wanxue Jiang.

Email: 1412520147@qq.com.

Author 8 - Chaoren Jiang.

Email: 2271482350@qq.com 